\title{
Dead in bed syndrome: Mystery and fear
}

\author{
Resham Raj Poudel, Nisha Kusum Kafle ${ }^{1}$, Bipin Belbase ${ }^{2}$, Pradip Kumar Kafle ${ }^{2}$ \\ Department of Medicine, Om Saibaba Memorial Hospital, ${ }^{1}$ Departments of Public Health and ${ }^{2}$ Medicine, Institute of Medicine, Kathmandu, Nepal
}

\section{A B S T R A C T}

Dead in bed (DIB) syndrome is a poorly understood cause of sudden and mysterious death in young people with type I diabetes on insulin therapy. Severe nocturnal hypoglycemia with unawareness is the most plausible mechanism which precipitates fatal arrhythmia in the vulnerable individual. Avoiding recurrent hypoglycemic episodes; screening for proarrhythmic condition in high-risk people with positive family history; and using real-time continuous glucose monitoring device in those with nighttime hypoglycemia and hypoglycemia unawareness can help to reduce the incidence of this deadly condition.

Key words: Arrhythmia, dead in bed syndrome, hypoglycemia, type I diabetes

\section{INTRODUCTION}

"Men fear most what they cannot see." - Henri Ducard (Batman Begins, 2005). Dead in bed (DIB) syndrome can be quite freaking to the patient, family and the physician. Someone with type 1 diabetes (T1D) in apparently good health; found DIB in the morning. ${ }^{[1]}$ What has happened? How and why? Was there anything that was overlooked or failed to foresee? Could it have been prevented? Many questions are left unanswered at the tragic event. DIB syndrome not only brings suffering in the family of the deceased; but also creates a bewildering psychosocial chaos and fear in all the children and the parents of children with diabetes.

\section{What IS DEAD IN BED SYNDROME?}

The term "DIB syndrome" was coined by Ian Campbell in an accompanying editorial in Diabetic Medicine in 1991; ${ }^{[2]}$ in which Tattersall and Gill described 22 cases of unexplained death in diabetics as DIB; in the analysis of 50 deaths of people with T1D, under 50 years of age,

\begin{tabular}{|l|l|}
\hline \multicolumn{2}{|c|}{ Access this article online } \\
\hline Quick Response Code: & Website: \\
\hline & www.joshd.net \\
\hline & \\
\hline & DOI: \\
\hline
\end{tabular}

which were considered sudden and unexpected in the UK. ${ }^{[1]}$ This first report was followed by a number of other surveys of mortality in young people with diabetes which confirmed the observation. ${ }^{[3,4]}$ DIB syndrome is a term used to describe sudden unexplained deaths in insulindependent young diabetic patients without any long term complications. The patients are found dead in or near an undisturbed bed, after having been in apparently good health the day before. No convincing cause of death is established; and the autopsy report is typically negative. ${ }^{[5]}$ A causality relationship of DIB syndrome with the use of human insulin had been talked about, ${ }^{[2]}$ but seems unlikely. ${ }^{[5]}$ The number of deaths from DIB syndrome is estimated to be 2-6/10,000 patient-years, which represents approximately $6 \%$ of all deaths in persons with diabetes aged $<40$ years. ${ }^{[5]}$

Clinical reports suggest that nocturnal hypoglycemia is a likely prerequisite of the event, but the sudden death is probably caused by cardiac arrhythmia. ${ }^{[6]}$ Arrhythmias are supposed to be more common during night-time hypoglycemia, possibly due to blunted nocturnal sympathoadrenal response and relatively increased parasympathetic activity. ${ }^{[7]}$ Genetic predisposition to long QT syndrome can put a person with diabetes at risk of a fatal arrhythmia. ${ }^{[8]}$ Malignant ventricular arrhythmia due to Brugada syndrome has also been postulated as a cause of sudden unexpected nocturnal death, since a glucose-insulin bolus can unmask the Brugada electrocardiographic signature in genetically

Corresponding Author: Dr. Resham Raj Poudel, Department of Medicine, Om Saibaba Memorial Hospital, Kathmandu, Nepal.

E-mail: poudelresham@gmail.com 
predisposed individuals. ${ }^{[9]}$ It is difficult to ascertain the diagnosis of hypoglycemia after death. ${ }^{[10]}$ However, in one case report of DIB syndrome, the patient was on a retrospective (non-real-time) sensor, and the sensor reading demonstrated glucose levels below $30 \mathrm{mg} / \mathrm{dl}$ $(1.7 \mathrm{mmol} / \mathrm{l})$ around the time of death; with $3 \mathrm{~h}$ of severe hypoglycemia $<40 \mathrm{mg} / \mathrm{dl},(2.2 \mathrm{mmol} / \mathrm{l})$, before death. ${ }^{[11]}$ Another report using sensor tracings has shown a lag time of 2-4 h before the onset of seizures when having severe hypoglycemia. ${ }^{[12]}$ If it is caused by severe hypoglycemia, why doesn't the person wake up? There has been a concern about autonomic neuropathy and the phenomenon of "hypoglycemia unawareness", which is basically a hypoglycemic episode without warning symptoms (tremors, palpitations, sweating from sympathetic activation) of the decreasing blood glucose level. Sleep, which naturally suppresses epinephrine, may also be a contributing factor. Evidence has been shown that hypoglycemic episodes per se precede the development of hypoglycemic unawareness; and hypoglycemic unawareness likely increases the risk of having a severe hypoglycemia. ${ }^{[13]}$

Psychosocial concerns of hypoglycemia and dead in bed syndrome

Although the mortality from DIB syndrome represents a small fraction of death, it needs timely discussion and review; not only from the clinical point of understanding the pathophysiology; but also as a tribute to the dead and the family of the dead who could never get an answer to what actually happened to their loved ones. An increase in the incidence of hypoglycemia seems likely today; when tight glycemic control is recommended for all patients with diabetes in order to prevent the long-term complications; and glycated hemoglobin $(\mathrm{HbA1c})$ targets are set lower than before. Hypoglycemia has been a possible cause of death ever since the discovery of insulin therapy. Its potential as a cause of sudden death is also highlighted by the early termination of the Action to Control Cardiovascular Risk in Diabetes (ACCORD) study due to increased mortality in the group that were intensively treated to lower HbA1c. ${ }^{[14]}$ It is one of the most difficult problems faced in the management of T1D. Frequent episodes can ruin the quality of daily living; cause seizures, coma and even death. The more dreaded forms, nocturnal hypoglycemia and the DIB syndrome are feared by many patients. That such recurrent attacks of nocturnal hypoglycemia might prove fatal elevates anxiety, depression, and anergia. ${ }^{[15,16]}$ Although the hypoglycemic symptoms warn the individuals to an impending episode, the warning signs can accentuate the fear of future hypoglycemia; eventually diminishing quality of life and reducing glycemic control. ${ }^{[17]}$ An event of hypoglycemia may elicit even greater fear in family members, ${ }^{[18]}$ and in non-diabetic spouses than in the patient himself. ${ }^{[19]}$ Such episodes may provoke marital conflict regarding diabetes management, ${ }^{[20]}$ and such experiences of stress and conflict can further derange glucose control. ${ }^{[21]}$ Ultimately, in an effort to prevent future hypoglycemic events, an individual may alter his behaviors (reducing or eliminating insulin dose, consuming high glycemic index food) that will eventually increase glucose levels. ${ }^{[17]}$

\section{Can we prevent dead in bed syndrome?}

Prediction and prevention of any event in health and disease rely on the understanding of its underlying pathophysiology. How can DIB syndrome be prevented depends on how much reliable are the theories that have been put forward till date. Since hypoglycemia is the most convincing assumption, avoiding nocturnal hypoglycemia seems the best intervention to prevent DIB.

\section{Prevention of nocturnal hypoglycemia}

Maintaining a balance in glucose control while avoiding hypoglycemia is the key to diabetes management. Hypoglycemia is a crucial issue for patients, their families and the physician. Diabetes education can provide patients with ways that will help to reduce fear and discourage choices that provide a rationale for poor glycemic control. ${ }^{[17]}$ Hypoglycemia begets hypoglycemia; ${ }^{[13]}$ and over time worsens to severe hypoglycemia, nocturnal hypoglycemia and arrhythmia. ${ }^{[7]}$ In T1D, the most common cause of hypoglycemia is a mismatch of insulin, food intake, and physical activity. To address these concerns, we need to provide patients and their families with a better understanding of the condition at every level. Mild to moderate hypoglycemic reactions include sweating, palpitations, tremor, hunger, nervousness which resolve within minutes of taking carbohydrates. That these warning symptoms are absent in severe hypoglycemia should be specifically taught to the patient and the family; along with the technique of treatment in the home setting with oral carbohydrates as well as glucagon injection if necessary. To help prevent severe hypoglycemia, there are several points to be taken into considerations:

- Taking the wrong type of insulin before going to bed can cause severe nighttime hypoglycemia. ${ }^{[22]}$ If a relatively large dose of bedtime basal insulin is replaced with a similar dose of regular or rapid-acting insulin, it can lower the blood glucose considerably and trigger a severe hypoglycemic reaction, which in turn can further precipitate a cardiac arrhythmia. ${ }^{[6-9]}$ Regimens using the newer basal insulin analoguesdegludec, detemir, glargine reduce the incidence of hypoglycemia, especially the severe and nocturnal hypoglycemia, compared to those using regular and 
neutral protamine hagedorn (NPH) insulin; ${ }^{[23,24]}$ while improving adherence to insulin therapy. ${ }^{[25]}$

- For physically active persons, we should focus on late post-exercise hypoglycemia. ${ }^{[5]}$ It is important to check glucose level after exercise, at bedtime and at 3:00 am if the activity is on or after late evening; in anticipation of possible hypoglycemia. If patients who are taking insulin increase their physical activity, they must either eat more or reduce the insulin dose before and after their activity; as an activity can cause hypoglycemia both during the activity and for several hours afterward. Late afternoon exercise can also cause nocturnal hypoglycemia. ${ }^{[26]}$ The dose of bedtime insulin should be decreased after more strenuous exercise, especially in the ones who do not regularly exercise.

- Patients on insulin who eat much less than usual need to reduce their insulin dose to prevent hypoglycemia.

- As the indiscriminate alcohol intake can cause severe hypoglycemia, its use should be restricted.

- In patients with T1D and a history of hypoglycemia, particularly who sleep alone; HbA1c targets should be more relaxed..$^{[5]}$ In persons with hypoglycemia unawareness, this will also allow the awareness to recover. ${ }^{[27]}$ If severe hypoglycemia occurs, the glucose targets should be raised immediately to prevent another episode.

- Those who have nighttime hypoglycemia, hypoglycemic unawareness, or a history of seizures, should be considered candidates for a real-time continuous glucose monitoring (CGM) device, which can be programmed to sound an alarm for readings below or above the target range. ${ }^{[28]}$ It helps to detect night-time glucose patterns and adjust insulin doses to avoid hypoglycemia. Such data from recent studies with continuous $\mathrm{CMG}$ are essential to convince all insurance carriers to reimburse this new life-saving technology.

- If patients who take insulin learn nothing else, they should learn to at least test their blood glucose, especially at bedtime.

- Early treatment of mild hypoglycemia must be emphasized so that progression to more severe consequences can be avoided.

- The diabetes educators should acquaint themselves more with the hazards of nocturnal hypoglycemia, so they will educate their patients to anticipate and prevent it.

\section{Prevention of arrhythmia}

Cardiac arrhythmia precipitated by hypoglycemia being considered the immediate cause of death in DIB syndrome; is likely another window of intervention to prevent such deaths. Prevention of deaths in arrhythmic syndromes is at times possible, when a correct diagnosis is available. Considering proarrhythmic events which occur in patients with diabetes, it may be useful to take a family history for sudden death in young or arrhythmic syndromes; and obtain at least a 12 lead electrocardiogram. ${ }^{[9]}$ As a standard practice ideal for any sudden unexplained natural death in the young, it is suggested to retain good quality deoxyribonucleic acid (DNA) following sudden death of a young person with diabetes. ${ }^{[29,30]}$ In cases, where a conclusive cause of death is not found, the family should be considered for referral to a cardiac genetic service, and genetic screening of autopsy DNA for cardiac ion channelopathies, particularly Brugada syndrome. ${ }^{[9]}$

\section{SUMMARY}

Hypoglycemia is a widely feared complication in T1D patients, which results from diet, physical activity or insulin mismatch; and tends to worsen with history of each preceding episode. Severe nocturnal hypoglycemia in the context of hypoglycemia unawareness is the most likely cause of the so called "DIB" syndrome presenting as an unexplained death in an undisturbed bed in a previously normal T1D person. The most convincing immediate cause of sudden death appears to be a hypoglycemia induced fatal arrhythmia. The value of insulin therapy in diabetes management and the importance of glycemic control in preventing long-term outcomes cannot be overemphasized. At the same time, hypoglycemia as a limiting factor to insulin therapy cannot be overlooked; so balance is the key. Balancing the insulin dose according to the changes in diet and physical activity helps in preventing sudden changes in glucose levels. In susceptible individuals, using long acting basal insulin analogues will prevent erratic changes in glucose levels, and reduce the episodes of unanticipated and severe nocturnal hypoglycemia. In very high risk individuals, using CGM can prove lifesaving.

\section{REFERENCES}

1. Tattersall RB, Gill GV. Unexplained deaths of type 1 diabetic patients. Diabet Med 1991;8:49-58.

2. Campbell I. Dead in bed syndrome: A new manifestation of nocturnal hypoglycaemia? Diabet Med 1991;8:3-4.

3. Thordarson $\mathrm{H}$, Søvik $\mathrm{O}$. Dead in bed syndrome in young diabetic patients in Norway. Diabet Med 1995;12:782-7.

4. Sartor G, Dahlquist G. Short-term mortality in childhood onset insulin-dependent diabetes mellitus: A high frequency of unexpected deaths in bed. Diabet Med 1995;12:607-11.

5. Sovik O, Thordarson H. Dead-in-bed syndrome in young diabetic patients. Diabetes Care 1999;22 Suppl 2:B40-2.

6. Weston PJ, Gill GV. Is undetected autonomic dysfunction responsible for sudden death in Type 1 diabetes mellitus? The 'dead in bed' syndrome revisited. Diabet Med 1999;16:626-31. 
7. Clark AL, Best CJ, Fisher SJ. Even silent hypoglycemia induces cardiac arrhythmias. Diabetes 2014;63:1457-9.

8. Tu E, Twigg SM, Semsarian C. Sudden death in type 1 diabetes: The mystery of the 'dead in bed' syndrome. Int $\mathrm{J}$ Cardiol 2010;138:91-3.

9. Skinner JR, Marquis-Nicholson R, Luangpraseuth A, Cutfield R, Crawford J, Love DR. Diabetic dead-in-bed syndrome: A possible link to a cardiac lon Channelopathy. Case Rep Med 2014;2014:647252.

10. Gormsen $\mathrm{H}$, Lund A. The diagnostic value of postmortem blood glucose determinations in cases of diabetes mellitus. Forensic Sci Int 1985;28:103-7.

11. Tanenberg RJ, Newton CA, Drake AJ. Confirmation of hypoglycemia in the "dead-in-bed" syndrome, as captured by a retrospective continuous glucose monitoring system. Endocr Pract 2010;16:244-8.

12. Buckingham $B$, Wilson DM, Lecher $T$, Hanas R, Kaiserman $\mathrm{K}$, Cameron F. Duration of nocturnal hypoglycemia before seizures. Diabetes Care 2008;31:2110-2.

13. Cryer PE. Hypoglycemia begets hypoglycemia in IDDM. Diabetes 1993;42:1691-3.

14. Gerstein HC, Miller ME, Byington RP, Goff DC Jr, Bigger JT, Buse JB. Action to Control Cardiovascular Risk in Diabetes Study Group. Effects of intensive glucose lowering in type 2 diabetes. N Engl J Med 2008;358:2545-59.

15. Strachan MW, Deary IJ, Ewing FM, Frier BM. Recovery of cognitive function and mood after severe hypoglycemia in adults with insulin-treated diabetes. Diabetes Care 2000;23:305-12.

16. McAulay V, Deary IJ, Ferguson SC, Frier BM. Acute hypoglycemia in humans causes attentional dysfunction while nonverbal intelligence is preserved. Diabetes Care 2001;24:1745-50.

17. Perlmuter LC, Flanagan BP, Shah PH, Singh SP. Glycemic control and hypoglycemia: Is the loser the winner? Diabetes Care 2008;31:2072-6.

18. Patton SR, Dolan LM, Henry R, Powers SW. Parental fear of hypoglycemia: Young children treated with continuous subcutaneous insulin infusion. Pediatr Diabetes 2007;8:362-8.

19. Gonder-Frederick L, Cox D, Kovatchev B, Julian D, Clarke W. The psychosocial impact of severe hypoglycemic episodes on spouses of patients with IDDM. Diabetes Care 1997;20:1543-6.
20. Cryer PE. Banting lecture. Hypoglycemia: The limiting factor in the management of IDDM. Diabetes 1994;43:1378-89.

21. Surwit RS, Schneider MS, Feinglos MN. Stress and diabetes mellitus. Diabetes Care 1992;15:1413-22.

22. Hanas R. Dead-in-bed syndrome in diabetes mellitus and hypoglycaemic unawareness. Lancet 1997;350:492-3.

23. Kalra S. Newer basal insulin analogues: Degludec, detemir, glargine. J Pak Med Assoc 2013;63:1442-4.

24. Goldman-Levine JD, Patel DK, Schnee DM. Insulin degludec: A novel basal insulin analogue. Ann Pharmacother 2013;47:269-77.

25. Kalra S, Unnikrishnan AG, Das AK. Improving adherence to insulin: Hope with degludec. J Soc Health Diabetes 2014;2:1-2.

26. Tsalikian E, Mauras N, Beck RW, Tamborlane WV, Janz KF, Chase HP, et al. Impact of exercise on overnight glycemic control in children with type 1 diabetes mellitus. J Pediatr 2005; 147:528-34.

27. Fanelli CG, Epifano L, Rambotti AM, Pampanelli S, Di Vincenzo A, Modarelli $F$, et al. Meticulous prevention of hypoglycemia normalizes the glycemic thresholds and magnitude of most of neuroendocrine responses to, symptoms of, and cognitive function during hypoglycemia in intensively treated patients with short-term IDDM. Diabetes 1993;42:1683-9.

28. Pitzer KR, Desai S, Dunn T, Edelman S, Jayalakshmi Y, Kennedy J, et al. Detection of hypoglycemia with the GlucoWatch biographer. Diabetes Care 2001;24:881-5.

29. Skinner JR, Duflou JA, Semsarian C. Reducing sudden death in young people in Australia and New Zealand: The TRAGADY initiative. Med J Aust 2008;189:539-40.

30. Ackerman MJ, Priori SG, Willems S, Berul C, Brugada R, Calkins $\mathrm{H}$, et al. HRS/EHRA expert consensus statement on the state of genetic testing for the channelopathies and cardiomyopathies this document was developed as a partnership between the Heart Rhythm Society (HRS) and the European Heart Rhythm Association (EHRA). Heart Rhythm 2011;8:1308-39.

How to cite this article: Poudel RR, Kafle NK, Belbase B, Kafle PK. Dead in bed syndrome: Mystery and fear. J Soc Health Diabetes 2015;3:22-5.

Source of Support: Nil. Conflict of Interest: None declared. 